Supporting Information

\title{
Gold(I) N-Heterocyclic Carbene and Carbazolate Complexes
}

Harrison M. J. Wang, Chandra Sekhar Vasam, Thomas Y. R. Tsai, Shih-Hua Chen,

A. H. H. Chang*, Ivan J.B. Lin*

Department of Chemistry, National Dong Hwa University, Shoufeng, Hualien 974,

Taiwan.

*Corresponding Authors

E-mail : ijblin@mail.ndhu.edu.tw and hhchang@mail.ndhu.edu.tw

Tel: 886-3-863-3599

Fax: 886-3-863-3570 
Table S1

Absorption Spectral Data of Au ${ }^{\mathrm{I}}-\mathrm{NHCs}$

\begin{tabular}{lc}
\hline Complex & $\lambda_{\max }\left(\mathrm{nm}, \mathrm{M}^{-1} \mathrm{~cm}^{-1}\right)$ \\
\hline$\left[\mathrm{Au}\left(\mathrm{Me}_{2}\right.\right.$-imy $\left.) \mathrm{Cl}\right]$ & $226(8300)$ \\
& $234(8900)$ \\
{$\left[\mathrm{Au}\left(\mathrm{Me}_{2}\right.\right.$-tazy $\left.) \mathrm{Cl}\right]$} & $247(11300)$ \\
& $244(4330)$ \\
{$\left[\mathrm{Au}\left(\mathrm{Me}_{2} \text {-imy }\right)_{2}\right] \mathrm{PF}_{6}$} & $220(10300)$ \\
& $233(15500)$ \\
& $244(15100)$ \\
{$\left[\mathrm{Au}\left(\mathrm{Me}_{2} \text {-tazy }\right)_{2}\right] \mathrm{PF}$} & $260(16300)$ \\
& $232(17400)$ \\
{$\left[\mathrm{Au}\left(\mathrm{Me}_{2}\right.\right.$-imy $\left.)(\mathrm{cbz})\right]$} & $255(4500)$ \\
& $353(3789)$ \\
{$\left[\mathrm{Au}\left(\mathrm{Me}_{2}\right.\right.$-tazy $\left.)(\mathrm{cbz})\right]$} & $370(3276)$ \\
{$\left[\mathrm{Au}\left(\mathrm{Me}_{2}\right.\right.$-bimy $\left.)(\mathrm{cbz})\right]$} & $349(3347)$ \\
& $367(2180)$ \\
& $360(5138)$ \\
& $369(2622)$
\end{tabular}

Absorption spectra of [Au(NHC)Cl] and [Au(NHC)2][PF6] were recorded in $\mathrm{CH}_{3} \mathrm{CN}$; for $[\mathrm{Au}(\mathrm{NHC})(\mathrm{cbz})]$ were recorded in $\mathrm{CH}_{2} \mathrm{Cl}_{2}$ 